\title{
Context-Aware Personalization Using Neighborhood-Based Context Similarity
}

\section{Abayomi Moradeyo Otebolaku \& Maria Teresa Andrade}

Wireless Personal Communications

An International Journal

ISSN 0929-6212

Wireless Pers Commun

DOI 10.1007/s11277-016-3701-2

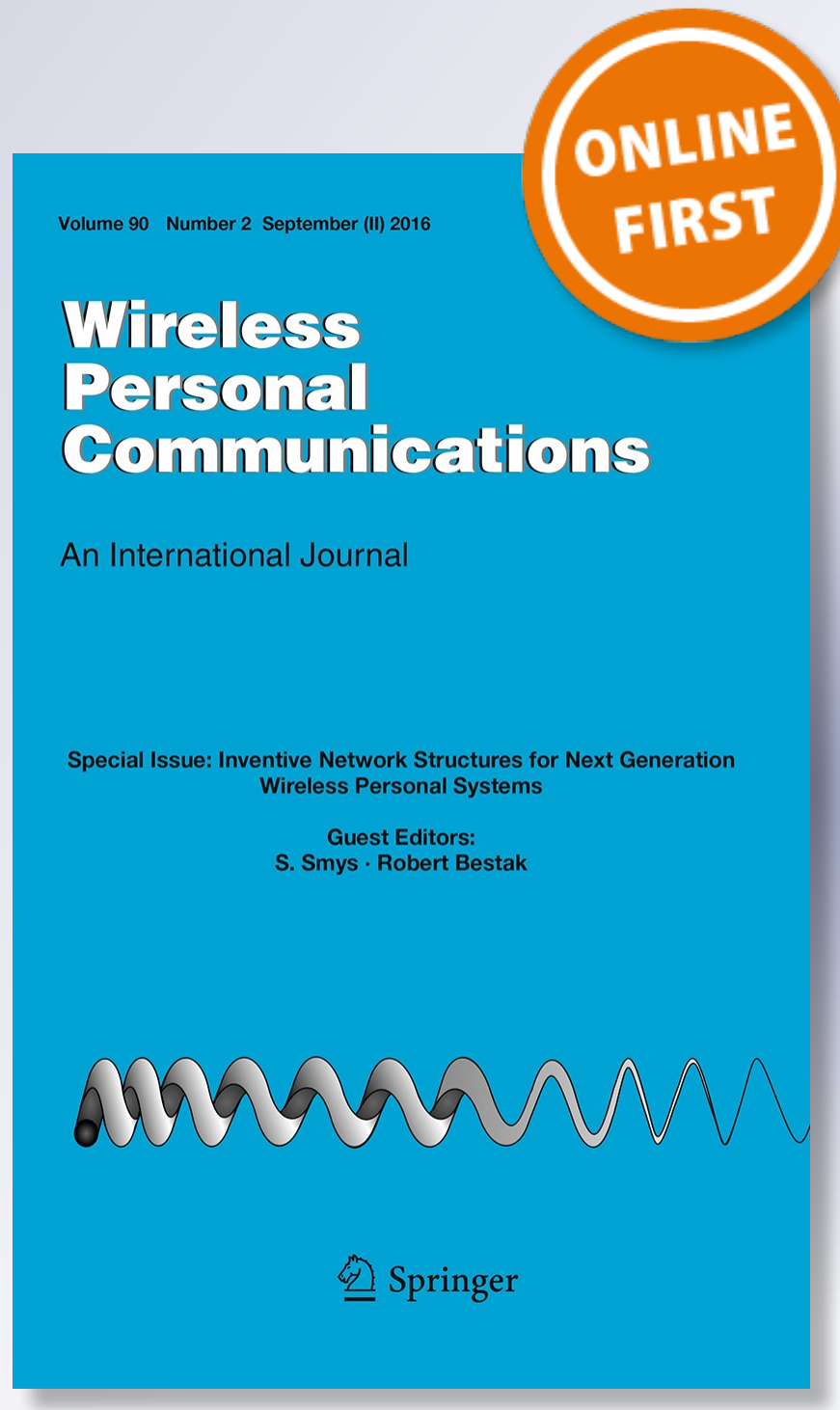

照 Springer 
Your article is protected by copyright and all rights are held exclusively by Springer Science +Business Media New York. This e-offprint is for personal use only and shall not be selfarchived in electronic repositories. If you wish to self-archive your article, please use the accepted manuscript version for posting on your own website. You may further deposit the accepted manuscript version in any repository, provided it is only made publicly available 12 months after official publication or later and provided acknowledgement is given to the original source of publication and a link is inserted to the published article on Springer's website. The link must be accompanied by the following text: "The final publication is available at link.springer.com". 


\title{
Context-Aware Personalization Using Neighborhood- Based Context Similarity
}

\author{
Abayomi Moradeyo Otebolaku ${ }^{1,2} \cdot$ Maria Teresa Andrade ${ }^{1,3}$
}

(C) Springer Science+Business Media New York 2016

\begin{abstract}
With the overwhelming volume of online multimedia content and increasing ubiquity of Internet-enabled mobile devices, pervasive use of the Web for content sharing and consumption has become our everyday routines. Consequently, people seeking online access to content of interest are becoming more and more frustrated. Thus, deciding which content to consume among the deluge of available alternatives becomes increasingly difficult. Contextaware personalization, having the capability to predict user's contextual preferences, has been proposed as an effective solution. However, some existing personalized systems, especially those based on collaborative filtering, rely on rating information explicitly obtained from users in consumption contexts. Therefore, these systems suffer from the socalled cold-start problem that occurs as a result of personalization systems' lack of adequate knowledge of either a new user's preferences or of a new item rating information. This happens because these new items and users have not received or provided adequate rating information respectively. In this paper, we present an analysis and design of a context-aware personalized system capable of minimizing new user cold-start problem in a mobile multimedia consumption scenario. The article emphasizes the importance of similarity between contexts of consumption based on the traditional k-nearest neighbor algorithm using Pearson Correlation model. Experimental validation, with respect to quality of personalized recommendations and user satisfaction in both contextual and non-contextual scenarios, shows that the proposed system can mitigate the effect of user-based cold-start problem.
\end{abstract}

Keywords Context-awareness $\cdot$ Personalization $\cdot$ Context similarity $\cdot$ Mobile users $\cdot$ Coldstart problem $\cdot$ Mobile devices

Abayomi Moradeyo Otebolaku

yomiotebolaku@gmail.com

1 Center for Telecommunications and Multimedia, INESC TEC, Faculty of Engineering, University of Porto, R. Dr. Roberto Frias, 4200-465 Porto, Portugal

2 Present Address: Department of Electronics, Telecommunications and Informatics, University of Aveiro, Campus Universitário de Santiago, 3810-193 Aveiro, Portugal

3 Faculty of Engineering, University of Porto, Porto, Portugal 


\section{Introduction}

The vast majority of mobile users are increasingly becoming frustrated and overwhelmed when deciding on the appropriate and relevant content to consume online. They are confused because of the unprecedented volume of multimedia content that is now available for online access via Internet and multimedia enabled devices. In addition, mobile users are overwhelmed because the content being pushed to them is often not relevant to their current contextual situations [1-5]. Therefore, the delivered content in most cases does not match their preferences. Imagine, for example, a recommendation system suggesting movies to a user while she is seated at her desk in the office! This kind of recommendation would be considered irrelevant or inappropriate considering the contextual situation of the user. Additionally, in searching for suitable content, users waste invaluable time and effort with no satisfying consumption experience. Thus, to adopt mobile devices as primary entertainment platforms for multimedia consumption, suitable technology is required to assist mobile users to obtain most relevant multimedia content from overwhelming volume of alternatives, matching their interests and contexts.

Personalized recommendation technologies and similar solutions providing this kind of functionality are common. For example, many massive online multimedia content providers have adopted and incorporated excellent recommendation services to provide their customers with effective and efficient suggestions [3]. However, some of these systems are designed to maximize business interests rather than user's interests, based on user provided item ratings [4]. These traditional approaches, as they are, cannot be used effectively in mobile environments because of cold-start problem [6-8]. The cold-start problem arises when personalization systems lack adequate knowledge of either the new user's preferences or of new items for providing relevant suggestions [6,7]. This often is due to the system's inability to gather sufficient information about users and the items they have preferred in the past [1]. Users normally prefer different content in different contexts. Thus, user preferences change as their contextual situations change. Traditional approaches, which are usually explored to address this problem, assume that user preferences are fixed and thus when user's context changes, such systems fail to provide relevant content matching the user's new contexts. For example, a user might prefer to listen to pop music when walking or jogging in her neighborhood. On the contrary, she might prefer to watch romantic movies while at home on rainy weekends! Therefore, traditional approaches that rely on explicit user ratings rather than on user's contextual information cannot be effectively applied. This problem reflects the fact that users cannot provide enough ratings in each context of consumption thereby resulting in cold-start problem [9].

To address this kind of cold-start problem, different techniques based on context-aware personalized recommendations have been proposed, assisting mobile users to obtain content according to their contextual preferences [1, 2, 9-23]. Figure 1 illustrates a typical architecture of a context-aware personalized recommendation system, exemplifying the system being proposed in this article. Thus, in this article we discuss in detail our proposal to address this new user problem using the similarity between a new user's contexts and those of other like-minded users to determine the new user's contextual preferences. The proposed solution uses contexts of a new user who does not have prior preferences represented in the system to identify like-minded users or her so-called neighbors rather than using explicit item or user ratings, which are prone to the cold-start problem [9]. We show that using context similarity using neighborhood algorithm to determine the so-called neighbors of the new user, it is possible to build a simple and yet effective context-aware personalized service that can provide relevant multimedia items to first time users based on 


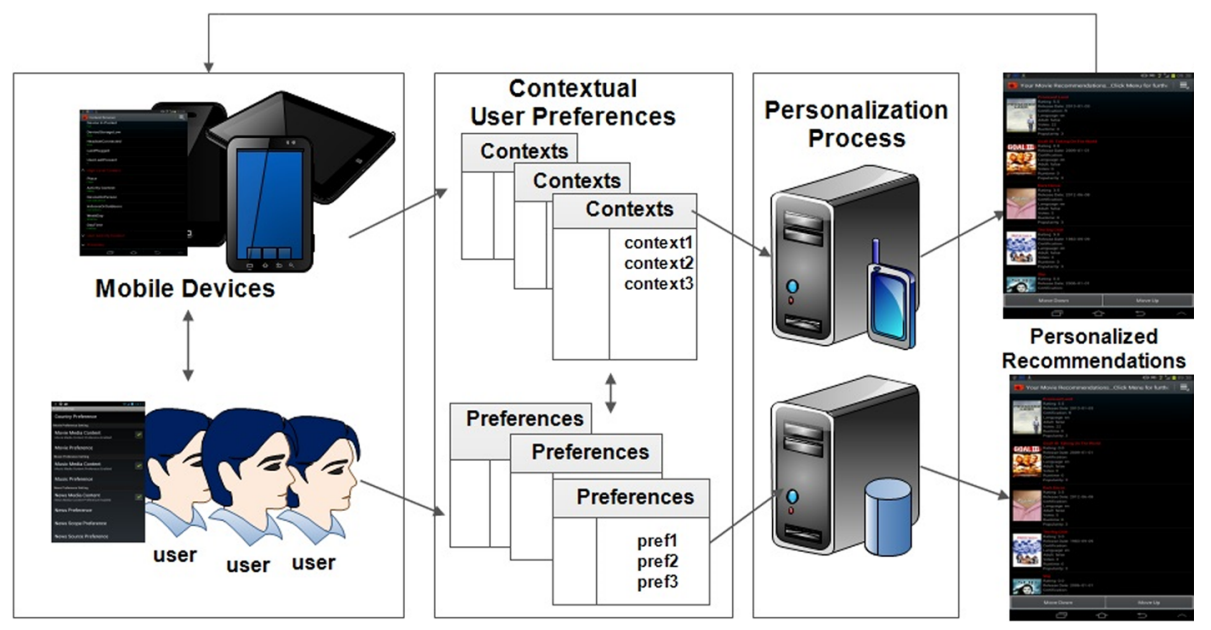

Fig. 1 A simplified architecture of a context-aware personalized recommendation system

three important requirements. First, such systems should be capable of determining the new user's current context [24]. Second, it should be able to use the identified contextual information to discover users who have been in the same or similar contexts, including the content they have consumed in those contexts. Third, and finally, it should be able to use the preferences of these newly identified neighbors to predict the preferences of the new user. With this approach and based on our experimental validation, we demonstrate that it is possible to mitigate the user-based cold-start problem using context similarity.

The rest of the paper is organized as follows. Section 2 presents an overview of existing work and how our proposal relates to and differs from them. In Sect. 3, detailed description of the proposed system is presented. In Sect. 4, the paper describes experimental evaluations of the system and discusses the results obtained. Finally, Sect. 5 draws some conclusion and provides the future direction of our work.

\section{Background and Related Work}

Conventional personalized recommendation systems have been designed to deliver multimedia content that matches user's preferences timely and effectively [2, 25]. However, these systems focus essentially on suggesting relevant items from millions of alternatives to users based on the assumption that user's tastes and preferences are fixed [5, 12]. They assist target users to explicitly discover relevant items using three popular methods namely: (1) evaluation of previously consumed multimedia content given by users, a process known as content-based filtering $(\mathrm{CBF}),(2)$ using evaluation of items consumed by like-minded users, otherwise known as collaborative filtering (CF) [7, 26, 27] and (3) using a combination of these methods in what is popularly called hybrid recommendations $[2,3,23]$. Although these conventional personalized recommendation techniques have been highly successful however, they are usually designed to address explicit recommendations of multimedia items. They anticipate explicit requests from users before providing recommendations using rating information provided by users or information about the content they have consumed. Thus, because there is no guarantee that users will 
always provide such rating information for multimedia content they consume, both $\mathrm{CF}$ and CBF suffer from the cold-start or new user problem [4, 6-9]. Therefore, these systems are not always reliable for providing effective recommendations to new users, i.e. those users that have never been provided with recommendations by the system. This problem is usually addressed by asking these new users to provide their preferences explicitly before they can be provided with recommendations [9]. Additionally, unlike collaborative-based recommendation systems, content-based recommendation systems suffer from overspecialization, which occurs when they provide users with only those items that are similar to those they have consumed in the past $[2,3,5,10,23]$. Furthermore, if a user has no record of consumption, it becomes difficult to provide such user with recommendations. To address these issues, hybrid recommendation based solutions were proposed. Whilst it addresses the weaknesses of both content-based and collaborative-based recommendations, hybrid recommendations profit from their strengths. Nevertheless, conventional hybrid recommendation proposals still cannot be effectively applied in mobile environments because they are designed based on the assumption that mobile user's preferences are fixed irrespective of their contextual situations [3-5].

In the last years, contextual recommendations have been proposed to address these weaknesses by taking into account the contextual situations in which users consume content $[2,9-12,19,20,23,28]$. These systems aim at using context-awareness to learn the preferences of users in different situations and then use the acquired knowledge to provide personalized recommendations. Comprehensive definition and information on how context information can be incorporated into recommendation systems have been presented by Adomavicius et al. [23]. Meanwhile, even though some existing proposals provide methods based on contextual information to recommend relevant information to users, these contextual approaches rely on explicit item or user ratings in specific contextual situations where users consume the content [3]. However, in mobile environments, users usually do not provide ratings every time they consume content $[3,5]$. Additionally, asking users to provide ratings when their context changes can only become a boring and annoying exercise. Thus, cold-start problem remains an issue in mobile environments despite being addressed by various researchers in different domains with excellent proposals.

One of such excellent proposals was published by Zhu et al. [29]. They proposed a generic context-aware recommendation system that takes as inputs user preferences, user contexts and device capabilities to provide multimedia content recommendations to smartphone users. The proposed system uses hybrid-based recommendation method, using vector space model (VSM) in combination with Bayesian and rule based algorithms to learn user's preferences and to generate recommendations. The system uses the Bayesian classifier to evaluate contextual information against media items consumed by users in those contexts and the rule based algorithm to determine the modality of the recommended content best matching the capability contexts of mobile devices. This system relies on user's contextual consumption history and not on context similarity to suggest new items.

Chen [12] also proposed a context-aware collaborative recommendation system that predicts user's preferences in different contexts. It extends traditional neighborhood-based collaborative recommendation system, using Pearson correlation, based on explicit ratings given by like-minded users in similar contexts. This work is similar to our proposal. However, rather than relying on explicit ratings obtained from users in previous contextual consumption, our work relies on predicting preferences of a new user by using similarity 
between her current contexts and those of other users who have consumed content in those given contexts.

Another interesting work by Pessemier et al. [11] uses daily activity contexts and other contextual information to provide personalized content according to the current activity a user performs, using collaborative recommendations. Also, a recent proposal by Alhamid et al. [14] uses social tags and user rating information to personalize multimedia content search in a context-aware collaborative recommendation system. In addition, it provides recommendations only if users make explicit requests. Nevertheless, using explicit rating information can lead to poor recommendation quality because in mobile environments, as earlier iterated, users usually do not, at all times, give ratings of content they have consumed.

Zhu et al. [30] proposed a comprehensive approach that first learns common contextaware preferences from the context logs of many users, representing the preferences of each user as a distribution of their common contextual preferences. They developed two techniques for mining common contextual preferences based on two different assumptions, namely context-independent and context-dependent assumptions fitting into different application scenarios. This work, however, did not focus on addressing cold-start problem but on how to use mobile users' context logs to predict their preferences.

More recently, Fernández-Tobías et al. [1] proposed an interesting solution to mitigate the cold-start problem based on (a) exploitation of personality information in a personalitybased $\mathrm{CF}$, to improve the recommendation prediction model, (b) personality-based active learning, utilizing personality information to identify additional useful preference data, elicited from users, in the target recommendation domain and (c) personality-based crossdomain recommendation that exploits personality information to better use preference data from auxiliary domains to compensate for the lack of user preference data in the target domain. This solution however, in addition to explicitly relying on rating information from users, did consider user's contextual information as an enabler for addressing cold-start problem.

In our previous work, we described how contextual information can be incorporated into content-based traditional recommendation systems via a novel user profiling approach to provide multimedia content recommendations [10]. However, in that project, similar to the content-based approach proposed by Wang et al. [22], we only used information about content consumed in the past by users to provide contextual recommendations in their current contextual situations. Thus, we did not focus on the cold-start problem but on the demonstration of how contextual information can be used to provide relevant recommendations based on the assumption that the system has sufficient consumption history of the target users.

The systems discussed above, even though some of them incorporate user's contextual information, they were designed based on explicit preference information from users in contexts where they consume multimedia content. Therefore, despite being excellent proposals however, they have not sufficiently addressed the cold-start problem. Thus, the new user problem remains a challenge with no unique solution that can be applied in any domain or situation [1]. In this paper, we demonstrate how similarity between contexts of new users and those of other users can be used to minimize the user-based cold-start problem with or without explicit rating information from users as proposed in previous solutions [10-12, 14, 16-22, 29]. 


\section{Context-Aware Personalization Using Context Similarity}

The proposed system was designed based on the traditional nearest neighbor collaborative recommendations using Pearson Correlation Coefficient (PCC) [12] to predict the target user's preference in the current context, according to the consumption experiences of her contextual neighbors as illustrated in Fig. 2. The contextual user's consumptions have been modelled using contextual profiles, consisting of contextual user preferences, which represent their consumptions in specific contexts. The user preferences are thus defined as follows:

$$
\begin{gathered}
u_{1}=p_{1 u_{1}, i_{1}, c_{1}}, p_{2 u_{1}, i_{2}, c_{2}}, \ldots, p_{n u_{1}, i_{n}, c_{m}} \\
u_{2}=p_{1 u_{2}, i_{1}, c_{1}}, p_{2 u_{2}, i_{2}, c_{2}}, \ldots, p_{n u_{2}, i_{n}, c_{m}} \\
u_{3}=p_{1 u_{3}, i_{1}, c_{1}}, p_{2 u_{3}, i_{2}, c_{2}}, \ldots, p_{n u_{3}, i_{n}, c_{m}} \\
\ldots \\
u_{k}=p_{1 u_{k}, i_{1}, c_{1}}, p_{2 u_{k}, i_{2}, c_{2}}, \ldots, p_{n u_{k}, i_{n}, c_{m}}
\end{gathered}
$$

where $u_{k}$ is user $\mathrm{k}\left(\mathrm{k}=1,2,3\right.$,.) and $p_{n u_{k}, i_{n}, c_{m}}$ represents her preference $p_{n u_{k}}$ for multimedia content $i_{n}$ in context $c_{m}$. We define a new user $U_{n}$, whose preference $p_{n u_{n}}$ for multimedia item $i_{n}$ in the context $c_{m}$ we want to predict.

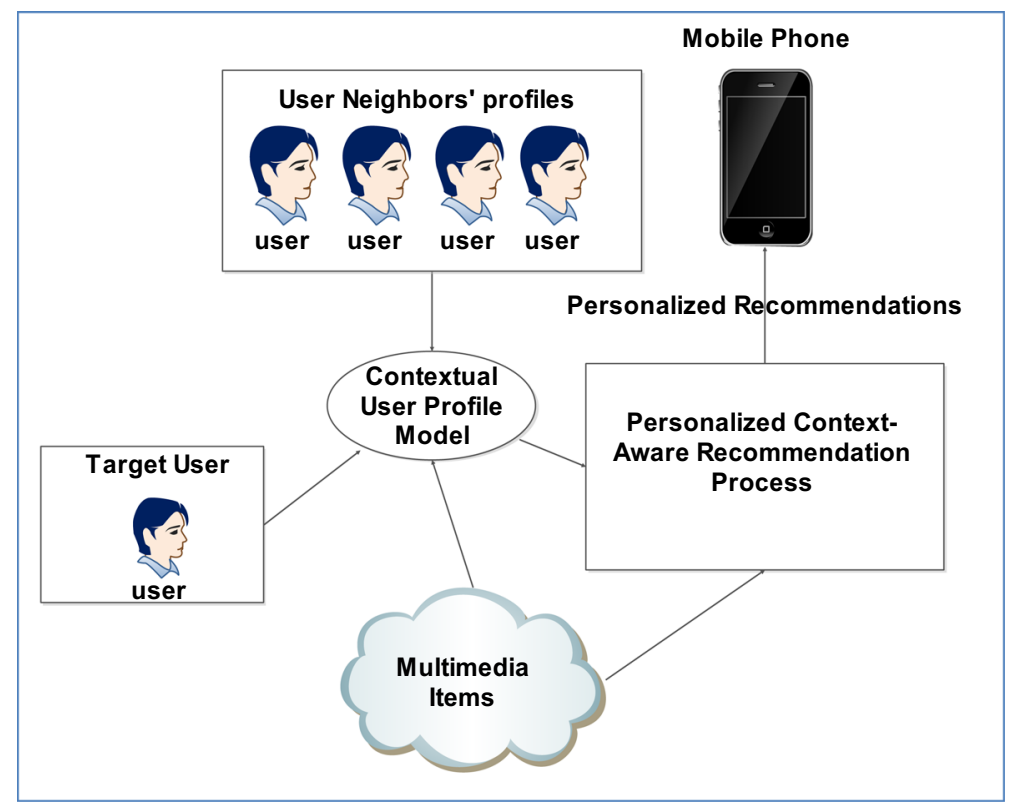

Fig. 2 Contextual user profiling and personalized recommendations 


\subsection{Learning Contextual User Profile}

Considering the problem definition in the last section, the proposed solution is built on a novel contextual profile incorporating contextual information in our existing recommendation system, consisting of users' consumption history. This information is obtained either explicitly or implicitly as described in our previous work [10, 20, 28]. In [10], we analyzed how contextual user profile model can be used in context-aware content-based recommendations. But here, we present an overview of the contextual user profile model and explain in the next section how we have extended it to utilize the similarity of users' contexts to predict preferences in a neighborhood(collaborative) process. As one of the key requirements to personalize multimedia content for mobile users, a contextual user profile learns the user's contextual preferences by representing the multimedia content a user consumes taking into consideration her contextual situations. In the user profile model, as illustrated in Fig. 3, the contextual situation represents information that characterizes a mobile user's consumptions, such as her activity, location, weather information, location illumination or noise level, time, etc. We define a mobile user as follows. $U=\left\{u_{1}, u_{2}, u_{3}\right.$, $\left.\ldots, u_{n}\right\}$ represents a set of $N$ mobile users. Each user $u_{k}$ is defined by a set of preferences $p_{n u_{k}}$ as illustrated in Eq. (4). Each preference $p_{n u_{k}}$ is defined by a set of category, $C a=\left\{c_{a 1}, c_{a 2}, c_{a 3}, \ldots, c_{a n}\right\}$ and each category is characterized by a set of genres, $G_{r}=\left\{g_{r 1}, g_{r 2}, g_{r 3}, \ldots, g_{r n}\right\}$. The category set represents the classifications of the consumed content, e.g. movie, news, music, etc. For example, movies are further characterized by genres such as action, drama, comedy, family, etc. It also includes a set of properties characterizing the content consumed by users at lower levels than the category and genre. These properties, defined by set $A=\left\{a_{1}, a_{2}, a_{3}, \ldots, a_{n}\right\}$, may include elements such as language, duration or year of publication of the content, etc. Additionally, we define a set of high-level contextual information, $C=\left\{c_{1}, c_{2}, c_{3}, c_{n}\right\}$ associated with each preference $\left(p_{n u_{k}}\right)$ of user $\mathrm{u}_{\mathrm{k}}$. In this definition, context $C$ has a relatively complex structure because many contexts can characterize the consumption of a user, reflecting the complexity of representing contextual profile concisely.

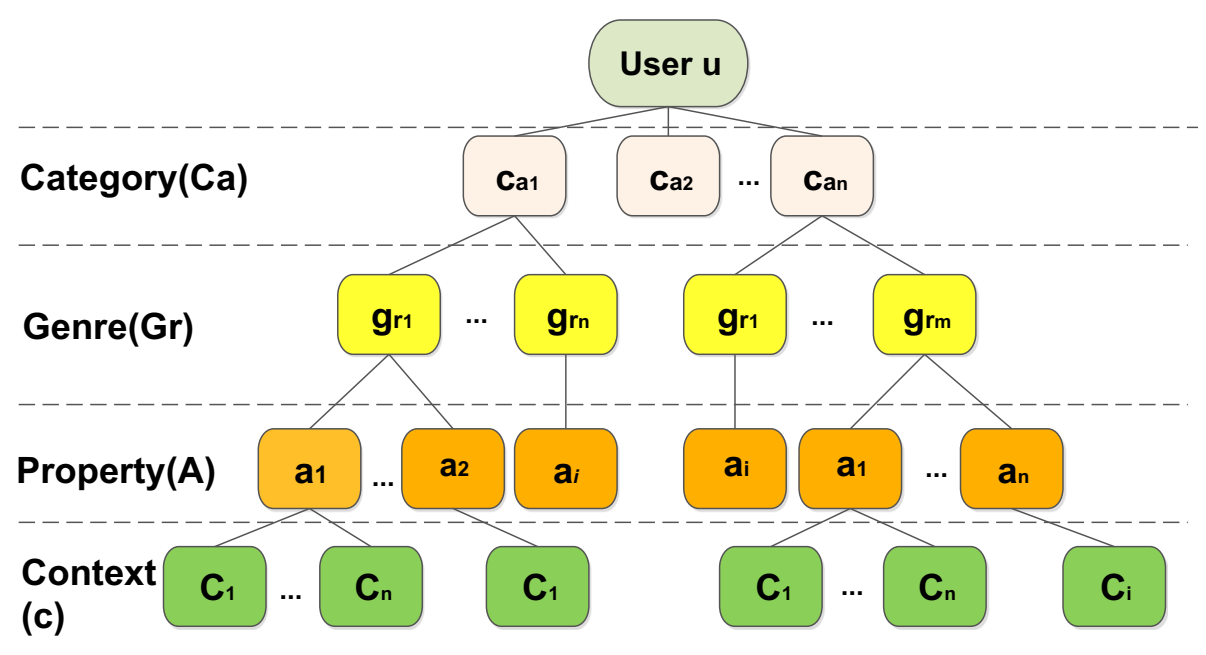

Fig. 3 Contextual user profile model showing its properties and how they relate to contextual information 
The user preference model is thus defined in a general form as follows.

$$
Y=f\left(x_{1}, x_{2}, x_{3}, \ldots, x_{n}\right)
$$

where $x_{1}, x_{2}, x_{3}, \ldots, x_{n}$ are user attributes and the preference information characterized by set $A, G_{r}$ and $C_{a} . Y$ is the dependent variable to be determined, and $f$ is the predictive function that can be learned via some classification model. For example, the system can predict the media item that user $u_{k}$ would like to consume using this model. However, the model defined by (5) does not account for contextual preferences of users but only defines the traditional user preferences. To incorporate contextual information into the model, we modify the above definition as follows.

$$
\mathrm{Y}=f\left(x_{1} c_{1}, x_{2} c_{2}, x_{3} c_{3}, \ldots, x_{n} c_{n}\right) \text { or } \mathrm{Y}=f c_{n}\left(x_{1}, x_{2}, x_{3}, \ldots, x_{n}\right)
$$

where $\mathrm{c}_{i}, \ldots, \mathrm{c}_{n}$ represent the contextual information and $f c_{n}$ is the function for identifying users' contextual preferences. This model can be used to learn the user profile in two modes. It works in the contextual mode and also in the non-contextual or traditional mode to determine the user preferences with and without contextual information respectively. The traditional mode (non-contextual mode) is executed in situations where user's contextual information is not available or when it is difficult or impossible to acquire. In this mode, an entire profile considering user consumption history (if any) is used. Alternatively, it could use the consumption history of users who are similar to the current user to learn the user's preference. The default mode is the contextual mode in which user's contextual information is used to learn the user's preferences. To illustrate the importance of the two models, in the evaluation in Sect. 4, personalized recommendation's Average Precisions (AP) and Mean Average Precision (MAP), based on these two models are compared.

To learn user's multimedia content consumption preferences based on the above profile model, the proposed system adopts the relevance feedback method [25]. Relevance feedback is a technique used to obtain a user's opinion on recommendations. It can speed up user preference learning process and improve the quality of recommendations [25]. There are two methods in literature used for learning user preferences based on user feedback namely, implicit and explicit relevance feedback [25]. In implicit user feedback, the system observes the content consumptions by the user and records information about the consumed content. In our case, such information includes category, genre, property, etc. of the multimedia content and most importantly the context in which users consume this content. Our implicit user feedback process, without asking for any information from the user, assigns a relevance value to the consumed content using the content metadata information as well as the context of consumption. The explicit user feedback requires asking user to provide ratings or some form of evaluation of the relevance of the consumed content, using metrics such as like or dislike of the recommended items. In mobile environments, this approach is obtrusive as it distracts and consequently bores users. This discourages users from using the recommendation system, which consistently requires them to provide rating information every time they consume content [25]. For this reason, we adopted an implicit user feedback method in combination with a simpler form of explicit user feedback. Our approach neither asks users for explicit rating information nor measure how long a user has spent during the consumption of a particular content, for example. Measuring time spent on recommended items as argued by Bjelica [25] may lead to wrong conclusions. Thus, rather than measuring time spent consuming content, we used a combination of contextual user profile learning model and the context in which users respond to recommendations either by clicking or not (the device's screen, for example) to 
learn the user's feedback on the recommended items. It then extracts implicit information about such items to learn whether users like or dislike them by assigning what we call relevance values to the extracted information, such as category of the content, its genre, etc. taking into consideration the contextual situation of the user at that given time. The system automatically assigns these relevance values in two numeric formats as illustrated in Eqs. (7) and (8) consisting of weight $w$ and lifetime $\gamma$ parameters.

$$
\begin{gathered}
w_{i}=(1-\alpha) \cdot w_{i-1}+\alpha \cdot \beta \cdot \gamma \\
\gamma=1-\left(\frac{t}{45}\right)^{5}
\end{gathered}
$$

The weight $w_{i}$ provides information on the number of times the user has consumed items of that category-genre-property in a specific context. The lifetime parameter provides an indication of the time elapsed since the last consumption occurred. Its value is set to 1 when the user consumes a content of that that belongs to a category-genre-property, and periodically decrements it if the subsequently consumed content does not belong to such category-genre-property. Smaller values indicate that the user has lost interest in that type of content, regardless of the value of its weight or contexts. In practice, it allows to give more importance to items consumed recently and less to those consumed long time ago. The factor $\alpha$ has a value in the range [0;1], assigning less or more importance to new category-genre-property. For newly created category-genre-property, $\alpha$ assumes the value of 1 ; otherwise its value, determined based on experiments, is 0.5 . The factor $\beta$ is the score given by the system and can assume the values of 1 or -1 . The value of 1 indicates that the user has consumed a new item having the characteristics as described by that categorygenre-property (i.e., indicates a preference of the user). The value of -1 is used to indicate that the user has rejected an item with those properties.

The lifetime parameter as explained earlier is set to 1 for matching items in the user profile, by assigning the value 0 to the factor $t$. The factor $t$ represents the number of days elapsed since the last time the user has consumed an item with the characteristics described by the profile. With Eq. (8), the relative importance of the category-genre-property of an item consumed by a user remains above 0.9 in the first 30 days after it has been visited, rapidly decreasing to zero after that period (non-negative values are automatically converted to zero) [10]. For all other category-genre-property in the user profile, the update of the lifetime parameter is performed by linearly increasing the value of $t$. This way, category-genre-property of a multimedia content that has been consumed before, but has not been seen or consumed for a long period will have either low or no impact on the user preferences evaluation.

\subsection{A High-Level View of New User's Preference Prediction Using Context Similarity}

The user profile model presented in the last section assumes that the system has adequate knowledge of those users represented by the model. Thus, for a new user, the model would provide poor recommendations.

To address this problem, we used contextual information and preferences of existing users as defined by the contextual user profile model (Eq. (6)) to predict the preferences of a new user considering her present context. 
To realize this process, the system first determines the new user's current contexts. This is possible because we have developed a dynamic context recognition model as an integral part of our broader personalization system [24, 28, 31]. First, the model acquires low-level context data from the user's handheld device to classify and identify their contextual situations. The detailed description of how this was realized is out of the scope of the present article, interested readers can take a look at Ref. [24] for details. Second, having identified the current context of the new user, the system then uses this context to search the profiles of existing users to identify those users with contexts that are similar to the new user's context. Those users with similar contexts as the current context of the new user are considered as her neighbors or friends. Third, by identifying these neighbors, the $n$ top most similar users are filtered, based on the order of magnitude of their contextual relevance to the new user. The profile of the user with the highest relevance value, as well as those of the other $n-1$ users, is used to compute the preference prediction for the new user. Figure 4 illustrates the work flow of the context-aware collaborative personalization. The process for identifying such relevant contexts and for predicting the new user's preference is described in the next section.

\subsection{Finding Users with Similar Contexts to the New User's Contexts}

The rationale behind finding users whose contexts are similar to the new user's context is to determine the content that would likely interest the new user in the current contexts based on the content consumed by those users with similar contexts. We designed the proposed solution based on the hypothesis that users with similar contexts usually share similar interests. Therefore, if we can establish the contextual situation of a new user, this

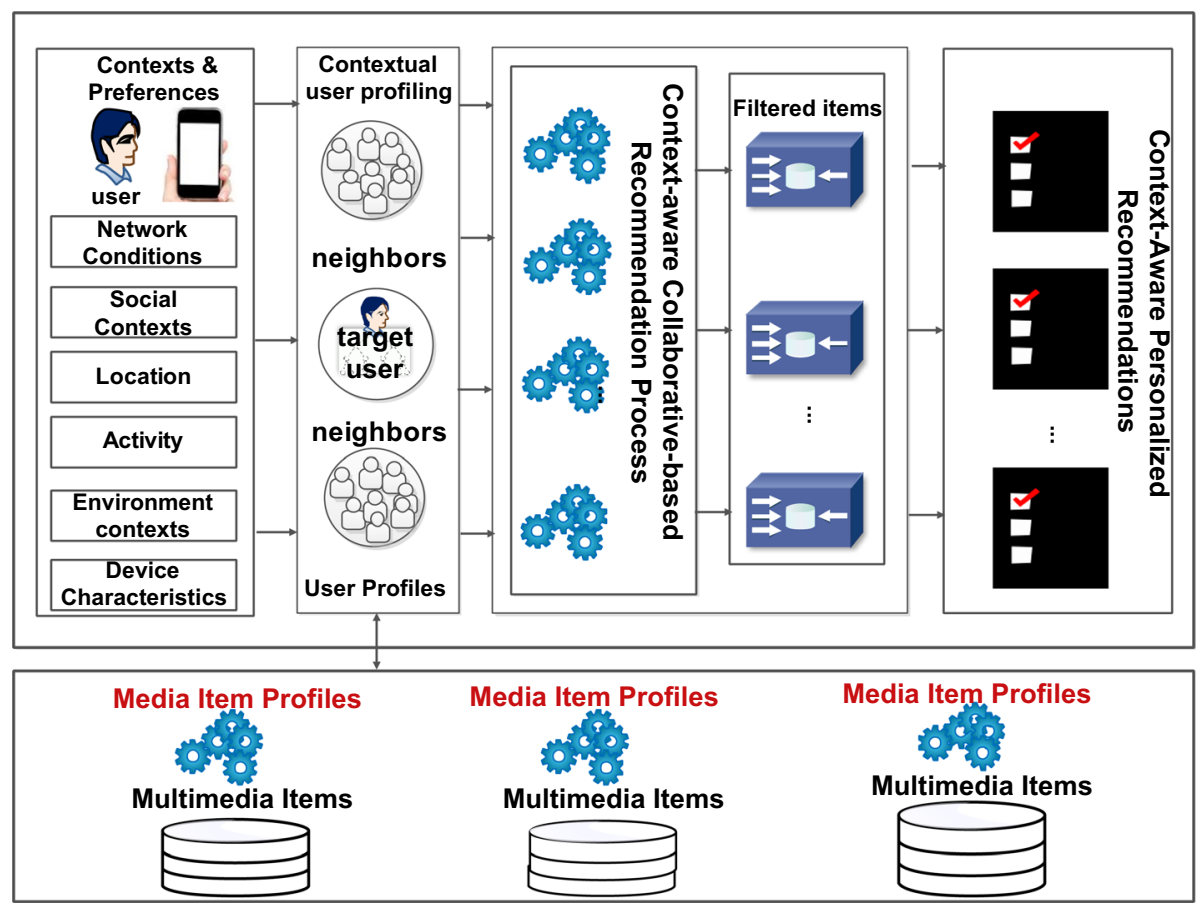

Fig. 4 The proposed context-aware collaborative personalization process workflow 
information can be used to identify users who prefer certain kinds of content in similar contexts. For example, when people gather at a cinema, it is most likely that they prefer movies of similar genres and properties, and thus in the future, movies or other similar content can be suggested. This hypothesis can be applied to restaurant or music recommendations. Users who are present at the same restaurants or concerts are most likely going to prefer similar menu or music respectively. Therefore, the similarity of contexts between a new user and existing users is an important information that can be used to predict the preferences of a new user in such contexts.

We assume, from the previous explanation, that contextual information has various types. For example, location type, time, activity type, etc. For each context type, we define a similarity function as used in [12]: $\operatorname{sim}_{n, m}\left(c_{n}, c_{m}\right)$. Where $c_{n}$ is the new user's current context and $c_{m}$ is the context of other users. In order to determine the similarity between a context and another, we define two classes of contexts. First, the categorical contexts that can assume unordered values, e.g. activity contexts with values such as Walking, Running, Jogging, etc. Second, the numeric contexts, which consist of ordered values such as temperature, noise level, illumination, etc.

To obtain the similarity between two numeric contextual preferences, we used (9).

$$
\operatorname{sim}_{n, m}\left(c_{n}, c_{m}\right)=\left\{\begin{array}{lll}
1-p & \text { if } & 0 \leq p \leq 1 \\
0 & \text { if } & p>1
\end{array}\right.
$$

where $p=\frac{\left|c_{n}-c_{m}\right|}{\text { Max-Min }}$, Max is the maximum numerical value of the context and Min is its minimum value. For example, let us take noise level as an example, if Min is $10 \mathrm{~dB}$, Max $=150 \mathrm{~dB}$, current, $c_{n}$ is 50 , and $c_{m}=60$ then the value of $\mathrm{p}$ is 0.0714 . In this case, the similarity between $c_{n}$ and $c_{m}$ is 0.93 .

To obtain similarity between two categorical features, a partial matching scheme similar to Lee and Lee [15] was adopted, using the domain knowledge. In this case, predetermined weighted scores are assigned to each categorical context. Tables 1, 2 and 3 illustrate examples of such weighted scores used as similarity values, which are automatically assigned by the system for matching activity, time and location context information. Contexts that match exactly are assigned 1.0, and those not matching maybe assigned less value or zero.

\subsection{Predicting New User Preferences}

In the last section, the article describes how to determine the similarity between a new user's current context and contexts of existing users. For all users with similar contexts, the

Table 1 Sample weighted scores for user activity contexts

\begin{tabular}{lllllll}
\hline Old user & \multicolumn{2}{l}{ New user } & & & & \\
\cline { 2 - 7 } & $\mathrm{W}$ & $\mathrm{R}$ & $\mathrm{J}$ & $\mathrm{St}$ & $\mathrm{S}$ & $\mathrm{L}$ \\
\hline Walking (W) & 1.0 & 0.2 & 0.3 & 0.1 & 0.1 & 0.1 \\
Running (R) & 0.2 & 1.0 & 0.5 & 0.2 & 0.3 & 0.1 \\
Jogging (J) & 0.3 & 0.5 & 1.0 & 0.1 & 0.3 & 0.0 \\
Standing (St) & 0.1 & 0.2 & 0.0 & 1.0 & 0.3 & 0.0 \\
Sitting (S) & 0.1 & 0.1 & 0.0 & 0.3 & 1.0 & 0.3 \\
Lying (L) & 0.0 & 0.0 & 0.0 & 0.0 & 0.2 & 1.0 \\
\hline
\end{tabular}


A. M. Otebolaku, M. T. Andrade

Table 2 Sample weighted scores for day of the week context information

Table 3 Sample weighted scores for location information

\begin{tabular}{llll}
\hline Old user & \multicolumn{2}{l}{ New user } & \\
\cline { 2 - 4 } & Weekday & Weekend & Holidays \\
\hline Weekdays & 1.0 & 0.1 & 0.2 \\
Weekend & 0.1 & 1.0 & 0.9 \\
Holidays & 0.1 & 0.9 & 1.0 \\
$\ldots$ & $\ldots$ & $\ldots$ & $\ldots$ \\
\hline
\end{tabular}

\begin{tabular}{llll}
\hline Old user & \multicolumn{2}{l}{ New user } & Home \\
\cline { 2 - 4 } & Cinema & Office & 0.0 \\
\hline Cinema & 1.0 & 0.0 & 0.0 \\
Office & 0.0 & 1.0 & 1.0 \\
Home & 0.0 & 0.0 & $\cdots$ \\
$\ldots$ & $\ldots$ & $\ldots$ & \\
\hline
\end{tabular}

system obtains the similarity values, computed using the strategies described in Sect. 3.3, in descending order of magnitude and selects the first $n$ users with the highest values. Among these $n$ users, consumption preferences of the user with the highest similarity, who we call a reference user and those of other $n-1$ users are then filtered to predict the preferences of the target (new) user. In this section, we explain the steps involved to realize this process.

The first step is to measure the correlation between each neighbor, designated as user $\mathrm{u}_{\mathrm{k}}$. We use the popular traditional Pearson coefficient to measure the correlation between consumption preferences of the neighbor user $u_{k}$ and each other neighbor $v_{k}[12,26]$. We designate her content preference in context $\mathrm{c}_{\mathrm{m}}$ for content $i$, having category-genreproperty as $p_{u_{k}, i_{n}, c_{m}}$, and that of the other neighbor as $p_{v_{k}, i_{n}, c_{m}}$. We use (10), which combines the preferences of neighbor $\mathrm{u}_{\mathrm{k}}$ and every other neighbor $\mathrm{v}_{\mathrm{k}}$ into a weighted average, using the correlations of these neighbors as the weights.

$$
p_{n}\left(u_{k}, v_{k}, c_{m}, i_{n}\right)=\frac{\sum_{i}\left(p_{u_{k}, i_{n}, c_{m}}-\overline{p_{u_{k}}}\right) \cdot\left(p_{v_{k}, i_{n}, c_{m},}-\overline{p_{v_{k}}}\right)}{\sqrt{\sum_{i}\left(p_{u_{k}, i_{n}, c_{m}},-\overline{p_{u_{k}}}\right)^{2} \sum_{i}\left(p_{v_{k}, i_{n}, c_{m},}-\overline{p_{v_{k}}}\right)^{2}}}
$$

where $\overline{p_{u_{k}}}$ and $\overline{p_{v_{k}}}$ denote the average of weights $\mathrm{w}$ defined in Eq. (7) for $\mathrm{u}_{\mathrm{k}}$ and $\mathrm{v}_{\mathrm{k}}$ respectively. $p_{n}\left(u_{k}, v_{k}, \mathrm{c}_{m}, i_{n}\right)$ returns the relevance of consumption preferences of both users in context $\mathrm{c}_{\mathrm{m}}$ for item $i_{n}$, containing category-genre-property.

Second, we defined a weighted preference $\left(p_{u_{k}, i_{n}, c}\right)$ of the reference user $\mathrm{u}_{\mathrm{k}}$ for an item $i$, which he consumed in contexts similar to the current context $c_{n}$ of the new user using context similarity $\operatorname{sim}_{n, m}\left(c_{n}, c_{m}\right)$ as weights. Because the context is multidimensional e.g. each location of the user is assumed to be characterized by, for example, the activity the user performs in that location, the location illumination, time of the day at the location, the day of the week, etc. the similarity of these context dimensions is computed and summed as illustrated in Eq. (11). $t$ in (11) is a normalizing factor such that the absolute values of the context similarity sum to unity. 


$$
p_{u_{k}, i_{n}, c}=t \sum_{C_{n} \in C} \sum_{t=1} \mathrm{p}_{u_{k}, i_{n}, c_{n}} \cdot \operatorname{sim}_{n, m}\left(c_{n}, c_{t}\right)
$$

Third, the preference of the new user is obtained using Eq. (12).

$$
p_{u_{n}, i_{n}, c_{n}}=\overline{p_{u_{k}, i_{n}, c_{m}}}+t \sum_{u=1}^{d}\left(\mathrm{p}_{u_{k}, i_{n}, c}-\overline{p_{u_{k}}}\right) \cdot p_{n}\left(u_{k}, v_{k}, c_{m}, i_{n}\right)
$$

where $d$ is the number of contextual neighbors of the new user and $t$ is a normalizing factor.

\section{Performance Evaluation}

In order to validate the proposed system, its experimental evaluation was conducted. We evaluated the ability of the system to provide relevant content to mobile users with inadequate information i.e. new users, using an Android based mobile application we have developed for suggesting contextual recommendations to mobile users [28]. We also evaluated the impact of neighborhood size on the quality of recommendations and recommendation time. Before delving into the details of the evaluations, we discuss the experimental data used as well as experimental setup.

\subsection{Experimental Data}

In the absence of suitable large-scale context-aware personalization data for our proposed system, we collected two sets of data for the experimental evaluation. The first set of data consists of over 4500 movie metadata obtained from the Movie Database (www. themoviedb.org), further enhanced using additional metadata retrieved from the Internet Movie Database (www.imdb.com). This metadata set contains 23 separate movie genres to form over 4500 multimedia content records. Each record contains, on the average, three different genre labels. Language, cast, country, duration, and release date serve as properties characterizing those genres. The Internet Movie Database provides API to access movie metadata from anywhere on the Web. Therefore, our system is able to access their massive repository of movie metadata for its recommendations.

The second set of data consists of contextual user data obtained from 200 anonymous volunteer users from the faculty of Engineering, University of Porto. The data represent category-genre-property of content, especially movies, consumed by these users in different contexts. About $80 \%$ of these volunteers are students, $9 \%$ are researchers, $2 \%$ are professors, $7 \%$ are professionals, and $2 \%$ are others. For experimental purposes, we only collected data on the genre of movies (category) consumed, the language, the country, etc. (as properties). At the same time, the respective contextual information of the consumed content was also collected from each user. Table 4 illustrates examples of context data associated with the collected user data. Note that in Table 4, C-G-P represents categorygenre-property of the content. These sets of data can be instantiated into the contextual user profile model deployed on the server side of the implementation to serve as the contextual consumption history. 
A. M. Otebolaku, M. T. Andrade

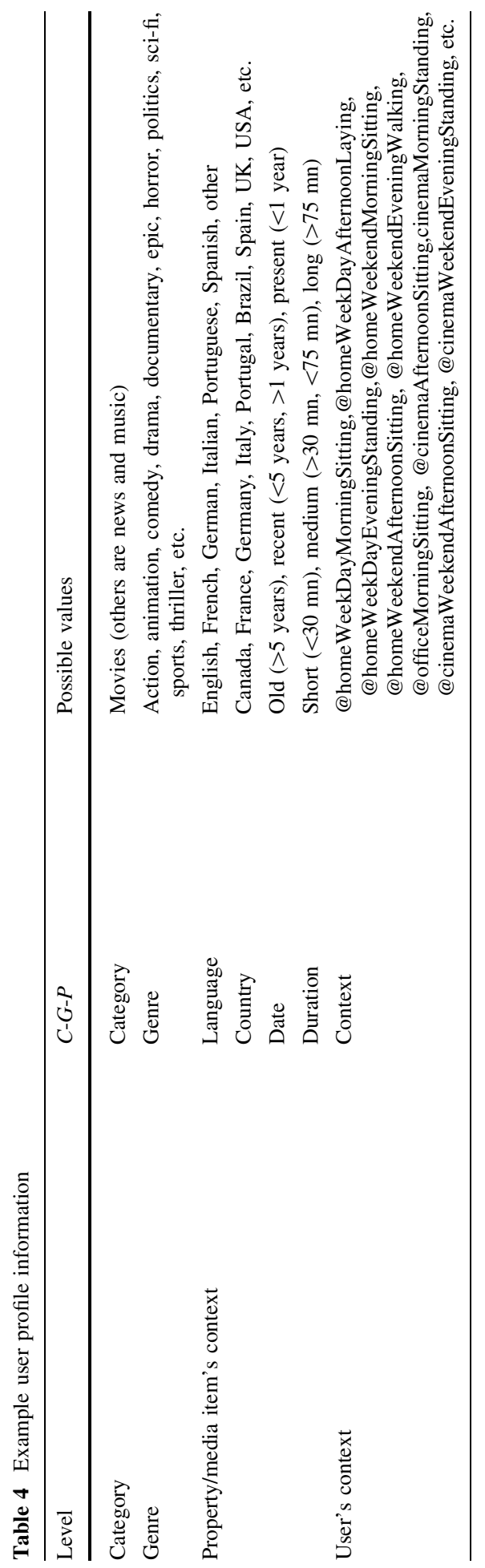




\subsection{Experimental Setup}

For evaluation purposes, we implemented the proposed solution as part of our existing Context-Aware Personalized Multimedia Recommendations (CAMR) application [28], consisting of a mobile client application running on handheld devices and a server application, both deployed in an experimental setup as illustrated in Fig. 5. The server application implements processes, including the contextual user preference model. We used these applications to provide contextual suggestions of content as illustrated in Figs. 6 and 7 , showing the implementation interfaces of the mobile client. The data described in the last section were instantiated in the server as contextual user profiles. Then for each user profile, and based on the contextual preferences they contain, recommendations were generated at 10 different times. The personalized multimedia content application receives contextual recommendation requests from the mobile client, it then processes the requests to determine the preferences of a target new user.

With this knowledge, the system determines the category-genre-property corresponding to those preferences, and then retrieves the set of content with similar genre and properties from online-based sources (e.g. theMovieDB, Youtube, IMDB, etc.) ranking them according to the preferences of the user. The top $\mathrm{k}$ items in the set are then provided as recommendations and displayed on the device screen. Users can then click to play preferred and relevant content.

As evaluation metrics, we decided to compute average precisions (AP@K) and mean average precisions (MAP@K) at top k recommendations as measures of quality of the personalized content generated for each recommendation and for all recommendations respectively [16].

Average Precision (AP@K) is the mean precision score obtained for each relevant item at top $\mathrm{k}$ recommendations in the test cases for every test user, computed as follows.

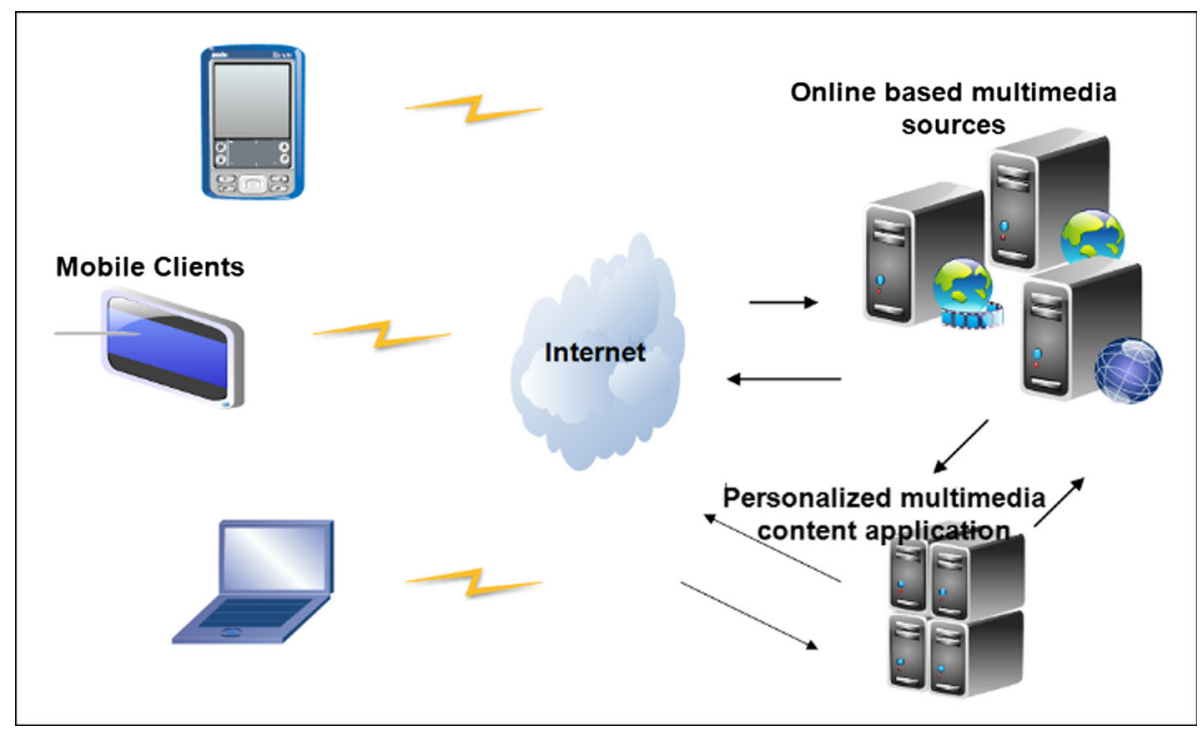

Fig. 5 Experimental evaluation setting 
Fig. 6 Recommendation process of the experimental application

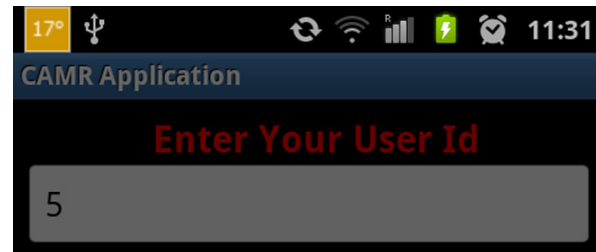

Recommend Movies

Recommend Similar Users

i Please wait...

Processing recommendations ...

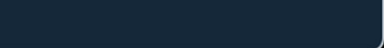

recuाIIIIEIIU Ivevvs

Recommend Movies

ContextServiceProvider prefs changed

$$
A P @ K=1 / m \sum_{i=1}^{m} \frac{1}{k_{i}}
$$

where $m$ is the number of relevant items and $\frac{1}{k_{i}}=0$ if item $i$ in the set is not relevant (not selected by the user as relevant). For these test users, recommendations were generated 10 times, with $\mathrm{k}=5$. This means each recommendation consists of a set of 5 items. Each time recommendation is generated for each test user, the average recommendation precision (AP@10) is computed for that user.

The mean average precision is given in (14), where $A P @ K$ represents the average precision at top $\mathrm{K}$ prediction results for test user $n$, and $\mathrm{N}$ denotes the total number of test users.

$$
M A P @ K=\frac{A P @ K}{|N|}
$$

\subsubsection{Evaluating New User Problems}

In the series of experiments conducted, the performance of the proposed system was evaluated for 20 test users. These 20 users were those with little or no information in the user profile model some of who we consider as new users. The validation was carried out in four carefully selected contextual situations, which we designated context types as follows: 
Fig. 7 Recommended items provided to users for evaluation

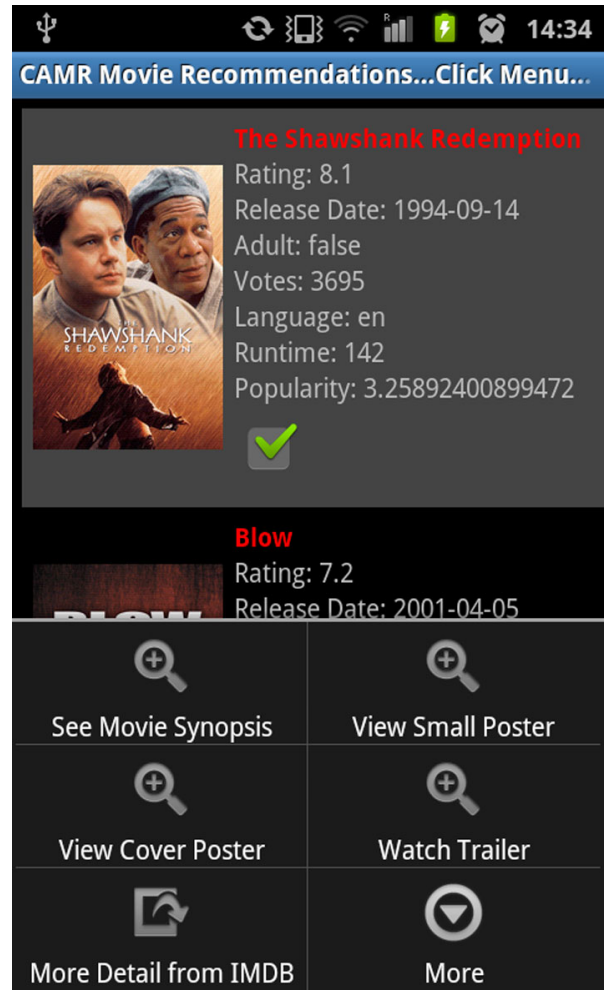

(A)@ @omeWeekendNightSitting,

(B)@ @inemaWeekendNightStanding,

(C)@homeWeekDayNightStanding,

(D) @ cinemaWeekDayNightSitting

(E)@noContext.

We also executed the experiments without using context information (No-Context) to compare performance of the model in those situations. We chose these contexts since it is not possible to evaluate the system under every possible context. Thus, it is obvious from the above context types that each consists of four contextual components. First, we have the location, second, third and fourth are the day of the week, time of the day and activity contexts respectively. Figure 8 shows the average precision of recommendations under each context type, and in no-context situations. The no-context situation is regarded as the baseline or the traditional personalized recommendation process. The most important conclusion from this figure is that in those four contextual situations (context types), the system's recommendation AP is higher than in no-context situations.

In addition, when we look at the number of recommendations, the quality of the context-based recommendations shows clearly that even at smaller number of recommendations, the system is able to provide better recommendations than in no-context recommendations. The low recommendation quality of the baseline evaluation underlines the cold-start problem, where the system could not find adequate information to predict the new user's preferences. 


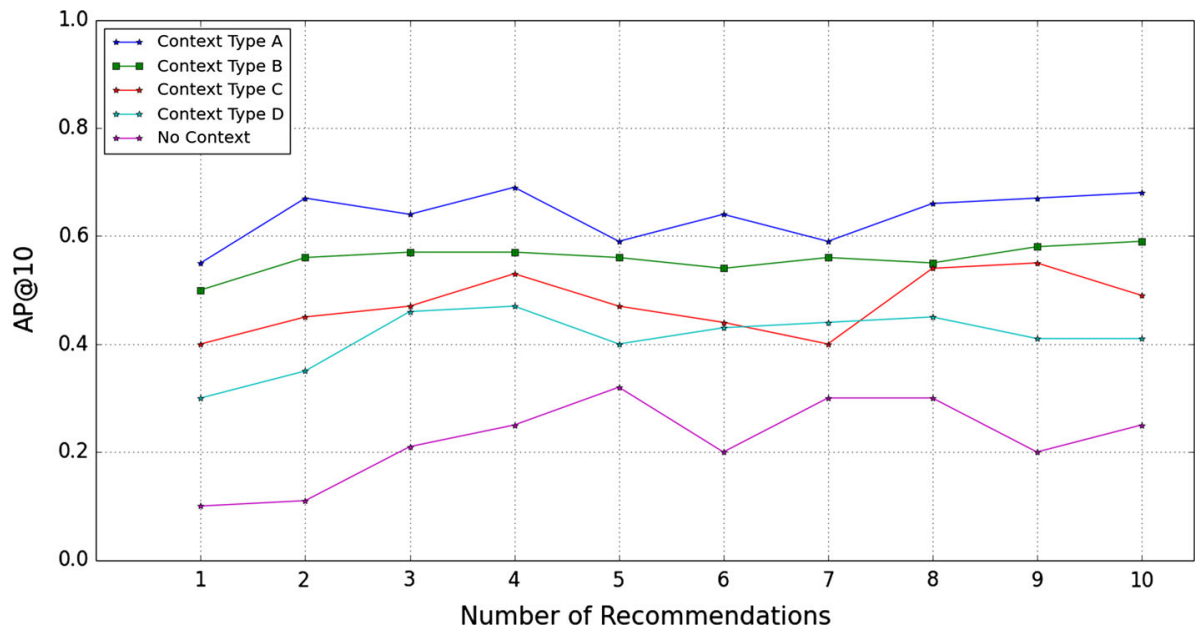

Fig.8 AP@10 of recommendations in selected contextual situations

The result further shows that the system can provide recommendations for users with little or no information as long as such new user's contexts can be determined and used in the recommendation process.

Furthermore, analyzing the AP under specific context types, @homeWeekendNightSitting $(A)$ and @ cinemaWeekendNightStanding $(B)$ tend to provide better recommendations, although the recommendations in all context types performed better than the non-context conditions. The reason is that naturally, and based on the contexts in which users have consumed movie items, the results suggest that more neighbors consumed movies in these two context types than in other context types. This explains why both context types produced slightly better performance than others as can be deduced from Fig. 8. To further explore the results, mean average precision (MAP@10) and their respective statistical confidence level (CL) were computed. Table 5 shows the CL for MAP@10 for each recommendation provided. The proposed solution is based on collaborative recommendation technique, where contexts of new users are utilized to identify those we refer to as their friends or other likeminded users. Thus, it can be argued that generally, the quality of contextual recommendations under those context types tends to improve with increasing number of recommendations.

This is a typical characteristic of collaborative recommendations, because the more interactions users have with the system, the more information it has to learn to predict the preferences of the users, and the better it is able to provide relevant recommendations. This explains why the recommendation's AP improved as the number of recommendations increased.

Table 5 Mean average precision and confidence level (confidence interval of @ 95\%) of AP@10

\begin{tabular}{llllll}
\hline Context type & A & B & C & D & E \\
\hline MAP@ 10 & 0.640 & 0.559 & 0.474 & 0.412 & 0.224 \\
CL & \pm 0.0287 & \pm 0.0155 & \pm 0.0318 & \pm 0.0308 & \pm 0.0446 \\
\hline
\end{tabular}




\subsubsection{Impact of Neighborhood Size on the Quality of Recommendations}

Although results of the previous experiments clearly show the advantage of using contextual information to minimize cold-start problem nevertheless, collaborative recommendations based on neighborhood methods require computation that grows with both user and item sizes [27]. We, therefore, decided to understand the influence of the number of neighbors on the recommendation quality and the profile processing time. Thus, another series of experiments were conducted, where we varied the values of the neighborhood size. Figure 9 shows the relationship between the neighborhood size and the corresponding recommendation AP for each context type used in the previous experiments. We observe that the number of users (neighbors) whose contexts are used to predict the preferences of a new user has significant impact on the average precision of the recommendations. However, the AP responds differently to different context types as the number of neighbors increases. The AP steadily increases as the number of neighbors increases for all the context types including the No-Context. Thus, considering this sensitivity of the AP of the recommendations for different neighborhood sizes, we consider neighborhood size between 10 and 20 as optimal, with average precision reaching up to $70 \%$. Figure 10 shows the neighborhood size and the profile processing time. Note that the time increases with the size of neighborhood. For example, the processing times for neighborhood sizes of 10,15 and 20 are 105, 180, and 310 (ms) respectively. The significance of this result is that the recommendation quality improves with increasing number of neighborhood size. Nevertheless, when compared with the results in Fig. 10, the profile processing time becomes a bottleneck because the recommendation time increased as we increased the neighborhood size.

\subsubsection{User-Based Evaluation}

In addition to evaluating the overall recommendation quality of the proposed solution, case study scenarios were set up in specific contextual situations in controlled experiments. The aim of the user-centered evaluation is to provide a generalized performance of the proposed solution involving real users. We evaluated user's satisfaction of the system to ascertain its usefulness and effectiveness of providing an understanding of how it would perform with actual users.

In the experiment, 20 individuals who were representatives of the system's target users constituted the test users, whereas 200 user profiles constituted the active contextual

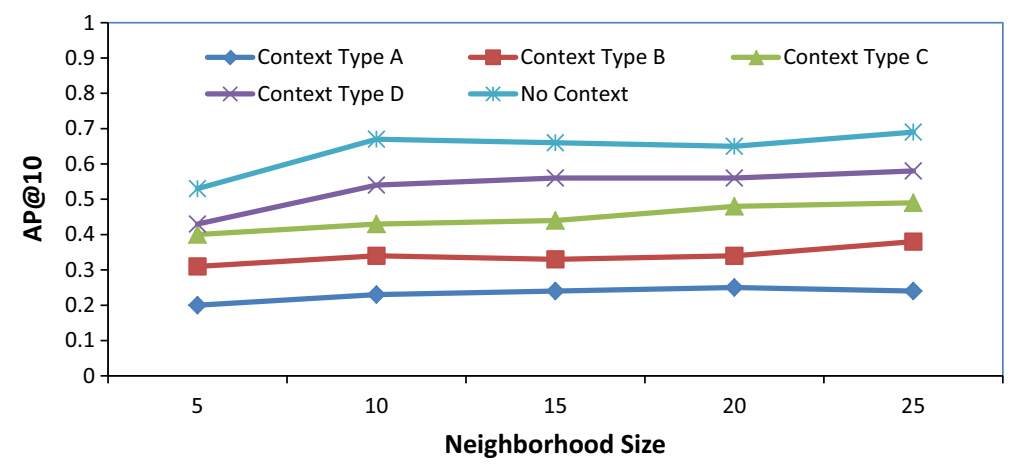

Fig. 9 Impact of neighborhood size on the AP@10 of recommendations for selected contextual situations 


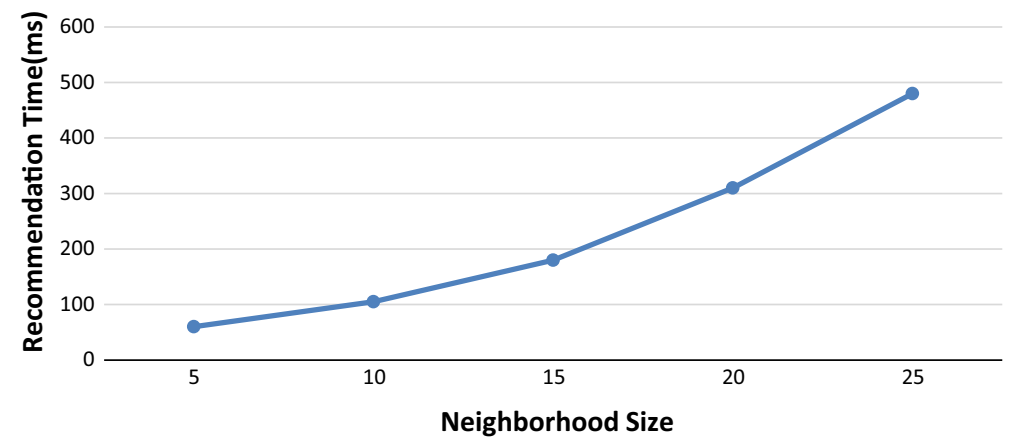

Fig. 10 Impact of neighborhood size on the AP@ 10 of recommendations for selected contextual situations

profiles in the server subsystem. Considering the difficulty of convincing people to participate voluntarily in this evaluation, we were not able to get all the 200 users whose contextual profiles were incorporated into the system to give feedback. However, judging by the number of users who participated in similar experiments and related work, we have a relatively high number of participants. For example, evaluation of the system presented by Pessemier et al. [11], had 16 participants. In [17] and [18], Oku et al. evaluated their systems with 5 and 8 participants respectively. Similarly, in [29] and [19] Yu et al. and Wang et al. evaluated similar systems based on 9 test users with 200 movie records and 10 test users respectively.

In the user evaluation process, we evaluated two contextual scenarios as our experimental case studies. The first case study is the home scenario where users usually perform activities characterized by other contexts. These contexts and their values are as follows. $\{$ Location $=$ Home,$\quad$ Activity $=$ Sitting,$\quad$ Day $=$ Friday,$\quad$ Time $=$ Afternoon,$\quad$ Illumina tion $=$ Bright, Noise level $=$ Normal $\}$. It then provides recommendations to users, based on this contextual information. The second case study is the office scenario where the system has to detect the user's activity and other contexts and use this information to provide recommendations for the users. The detected contexts are $\{$ Location $=$ Office, Activity $=$ Sitting, $\quad$ Day $=$ Monday,, Time $=$ Afternoon, Illumination $=$ Bright,$\quad$ Noise level $=$ Normal $\}$. In both scenarios, the users then provide a satisfaction score for each recommendation. For example, in each scenario, 10 recommendations were provided in decreasing order of relevance to the user's contextual situations.

Test users were asked to evaluate the degree of satisfaction of the recommendations on a 5-point scale, where "5", the maximum point, means very satisfied and " 1 ", the minimum point, means not satisfied. We analyzed the feedback provided by these users. We converted the satisfaction scores into binary values as follows. All scores between 3 and 5 were considered satisfactory; those below 3 were considered not satisfactory. We collected all the scores and then calculated the average to obtain the overall satisfaction from the user's perspectives. The overall satisfaction obtained was $69 \%$. Additionally, the final feedback from the test users was analyzed and used to compute average precisions for each test user based on top 10 recommendations. The mean average precision (MAP) obtained was 0.65 , which shows that the proposed system can provide recommendations that are relevant to each user in specific contexts with confidence level of $0.65 \pm 0.059(95 \%$ confidence interval), thereby minimizing the number of false positives. 


\section{Conclusion}

This article presents a detailed design of a context-aware personalization system that uses neighborhood-based approach and contextual information to minimize the impact of coldstart problem, which is common in conventional personalization systems. The proposed solution focuses on the delivery of personalized mobile content to handheld devices.

The main contribution of this article is the use of contextual information to identify likeminded users or neighbors whose preferences or those similar would interest a new user. We argue that in similar contextual situations, users tend to express similar preferences for content consumption. The contextual information of the new user is determined dynamically using our existing context recognition system [31]. Essentially, after identifying these users, their contextual preferences are then used to predict preferences for the new user.

Experiments conducted to validate the proposed system show that using similarity between contextual information, the cold-start problem can be minimized with or without rating information. Evaluation under carefully selected contextual situations showed consistently improved average recommendation quality. On the contrary, evaluation of recommendations under no-context condition showed poor performance, reflecting the cold-start problem. Thus, when we compared the results of recommendations under contextual situations with those under no-context, the contextual recommendations showed clearly, how context can be explored to address the cold-start problem, which is obvious in the results as contextual recommendations produced better precision of up to $70 \% \mathrm{com}$ pared to that of no context that produced poor precision between $10 \%$ and $43 \%$.

In the user evaluation process, based on two contextual case studies, the results obtained also confirm that contextual information can be used to improve the relevance of content suggestions with or without collecting rating information from user every time content is consumed. Further experimental tests on the impact of the neighborhood size on the recommendation AP clearly showed improved quality with increasing number of neighbors. However, in another series of tests to evaluate the impact of the neighborhood size on the user profile processing time, we observed that the larger the size of the neighbors, the longer time it takes to process user information. Therefore, there is a tradeoff between higher quality of the recommendations and processing time. Higher APs due to larger neighbor size means longer processing time. Finally, on the basis of the above results, we conclude that context similarity can be used to effectively mitigate the effects of userbased cold-start problem.

In the future, we plan to investigate alternative methods to improve the recommendation quality and processing time that tend to deteriorate with increasing number of neighbors. We would also like to investigate some other advanced and robust collaborative approaches, such as combining neighborhood and latent factor models in a context-aware collaborative recommendation process. The latent factor approaches, such as Singular Value Decomposition (SVD), comprise alternative methods for collaborative filtering with better ability to uncover latent features for better prediction of user's observed preferences $[13,21]$.

Acknowledgments This work was partly financed by the Portuguese Fundação para a Ciência e a Tecnologia (FCT) with grant SFRH/BD/69517/2010. The authors would also like to thank all the online participants who voluntarily participated in the user profile data collection and evaluation of the system. 


\section{References}

1. Fernández-Tobías, Braunhofer, I. M., Elahi, M., Ricci, F., \& Cantador, I. (2016). Alleviating the new user problem in collaborative filtering by exploiting personality information. User Modeling and UserAdapted Interaction, 22(2), 221-255.

2. Adomavicius, G., \& Tuzhilin, A. (2005). Towards the next generation of Recommender Systems: A survey of the State-of-the-art and Possible Extensions. IEEE Trans on Knowledge and Data Engineering, 17(6), 734-749.

3. Xia, F., Asabere, N. Y., Ahmed, A. M., Li, J., \& Kong, X. (2013). Mobile multimedia recommendation in smart communities: A survey. IEEE Access, 1, 606-624.

4. Martin, F. J., Donaldson, J., Ashenfelter, A., Torrens, M., \& Hangartner, R. (2011). The big promise of recommender systems. AI Magazine, 32(3), 19-27.

5. Yujie, Z., \& Licai, W. (2010). Some challenges for context-aware recommender systems. In Proceedings of workshop on recommender systems at the 5th international conference on computer science and education. IEEE (pp 362-365).

6. Son, L. H. (2016). Dealing with the new user cold-start problem in recommender systems: A comparative review. Information Systems, 58, 87-104.

7. Lika, B., Kolomvatsos, K., \& Hadjiefthymiades, S. (2014). Facing the cold-start problem in recommender systems. Expert Systems with Applications, 41(4 PART 2), 2065-2073.

8. Kwon, H. J., \& Hong, K. S. (2011). Personalized smart TV program recommender based on collaborative filtering and a novel similarity method. IEEE Transactions on Consumer Electronics, 57(3), 1416-1423.

9. Krstic, M., \& Bjelica, M. (2012). Context-aware personalized program guide based on neural network. IEEE Transactions on Consumer Electronics, 58(4), 1301-1306.

10. Otebolaku, A. M., \& Andrade, M. T. (2014). Context-aware user profiling and multimedia content classification for smart devices. In 28th IEEE international conference on advanced information networking and applications workshops (WAINA), (pp. 560-565).

11. Pessemier, T. D., Dooms, S., \& Martens, L. (2014). Context-aware recommendation through context and activity recognition in a mobile environment. Multimedia Tools and Applications, 72(3), 2925-2948.

12. Chen, A. (2005). Context-aware collaborative filtering system: Predicting the user's preference in the ubiquitous computing environment. Location- and context-awareness (pp. 244-253). Berlin: Springer.

13. Koren, Y. (2008). Factorization meets the neighborhood: A multifaceted collaborative filtering model. In ACM SIGKDD (pp. 426-434).

14. Alhamid, M. F., Rawashdeh, M. H., Osman, A. L., Hossain, M. S., \& El Saddik, A. (2015). Towards context-sensitive collaborative media recommender system. Multimedia Tools and Applications, 74, 11399-11428. doi:10.1007/s11042-014-2236-3.

15. Lee, J., \& Lee, J. (2008). Context awareness by case-based reasoning in a music recommendation system. Ubiquitous Computing Systems, 45-58.

16. Zhu, H., Chen, E., Xiong, H., Yu, K., Cao, H., \& Tian, J. (2014). Mining mobile user preferences for personalized context-aware recommendation. ACM Transactions on Intelligent Systems and Technology, 5(4), 1-27.

17. Oku, K., Nakajima, S., Miyazaki, J., \& Uemura, S. (2006). Context-aware SVM for the context dependent information recommendation. In Proceedings of the 7th international conference on mobile data management (p. 109).

18. Oku, K., Nakajima, S., Miyazaki, J., \& Uemura, S., Kato, H. \& Hattori, F. (2010). A recommendation system considering users' past/current/future contexts. ids.csom.umn.edu, pp. 3-7, [Online].

19. Wang, X., Rosenblum, D. \& Y. Wang, Y. (2012). Context-aware mobile music recommendation for daily activities. In Proceedings of the 20th ACM international conference on multimedia, October 29November 02, 2012, Nara, Japan.

20. Otebolaku, A. M., \& Andrade, M. T. (2015). Context-aware media recommendations for smart devices. Journal of Ambient Intelligence and Humanized Computing, 6(1), 13-36. doi:10.1007/s12652-0140234-y.

21. Alhamid, M. F., Rawashdeh, M., Dong, H., Hossain, M. A., \& Saddik, A. E. (2016). Exploring latent preferences for context-aware personalized recommendation systems. IEEE Transactions on HumanMachine Systems, 46(4), 615-623. doi:10.1109/THMS.2015.2509965.

22. Wang, Z., Yu, Z., Zhou, X., Chen, C., \& Guo, B. (2016). Towards context-aware mobile web browsing. Wireless Personal Communications. doi:10.1007/s11277-016-3454-y. 
23. Adomavicius, G., Tuzhilin, A., Sankaranarayanan, R., \& Sen, S. (2005). Incorporating contextual information in recommender systems using a multidimensional approach. ACM Transaction on Information Systems, 23(1), 103-145.

24. Otebolaku, A. M., \& Andrade, M. T. (2016). User context recognition using smartphone sensors and classification models. Journal of Network and Computer Applications, 66(B), 33-51.

25. Bjelica, M. (2011). Unobtrusive relevance feedback for personalized TV program guides (2011). IEEE Transactions on Consumer Electronics, 57(2), 658-663.

26. Desrosiers, C., \& Karypis, G. (2011). A comprehensive survey of neighborhood-based recommendation methods. In: F. Ricci, L. Rokach, B. Shapira \& P. B. Kantor (Eds.), Recommender systems handbook (pp. 107-144). New York: Springer.

27. Sara, B. M., Karypis, G., Konstan, J. \& Riedl, J. (2001). Item-Based collaborative filtering recommendation algorithms. $10^{\text {th }}$ International World Wide Web Conference, ACM Press, pp. 285-295.

28. Otebolaku, A. M., \& Andrade, M. T. (2014). A context-aware framework for media recommendations on smartphones. In L. De Strycker (Ed.) Proceedings of the European conference on the use of modern information and communication technologies (ECUMICT 2014), Gent, March 2014. 27th-28th of March 2014, Gent, Belgium, Published by Springer Lecture Notes in Electrical Engineering (Vol. 302, pp. 87-108).

29. Yu, Z., Zhou, X., Zhang, D., Chin, C. Y., Wang, X., \& Men, J. (2005). Supporting context-aware media recommendations for smartphones. IEEE Pervasive Computing, 5(3), 68-75.

30. Zhu, H., Chen, E., Xiong, H., Yu, K., Cao, H., \& Tian, J. (2014). Mining mobile user preferences for personalized context-aware recommendation. ACM Transactions on Intelligent Systems and Technology, 5(4), 1-27.

31. Otebolaku, A. M., \& Andrade, M. T. (2013). Recognizing high-level contexts from smartphone built-In sensors for mobile media content recommendation. In 14th IEEE international conference on mobile data management (MDM) (pp. 142-147).

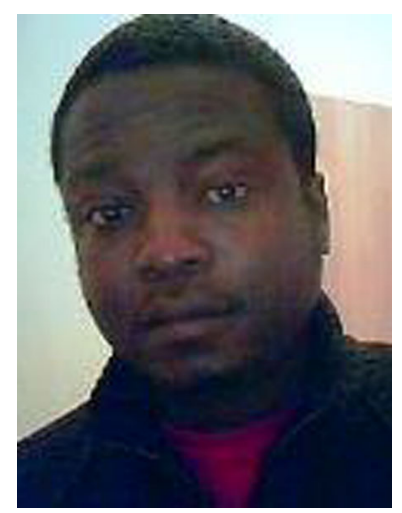

Abayomi Moradeyo Otebolaku received his Ph.D. in Telecommunications Engineering from the Faculty of Engineering, University of Porto, Portugal. He was a research engineer at the Center for Telecommunications and Multimedia, INESC TEC, Porto. He was also a recipient of Portuguese government's Fundaçao para a Ciência e a Tecnologia - Foundation for Science and Technology (FCT) doctoral grants (Bolsa de Doutoramento). He is currently a Postdoctoral Associate at the Department of Electronics, Telecommunications and Informatics, University of Aveiro, Portugal. Dr Otebolaku is a member of the Institute of Electrical and Electronics Engineers (IEEE). His primary research interests are in the areas of context-aware computing, smartphone sensing and contextaware personalization. 


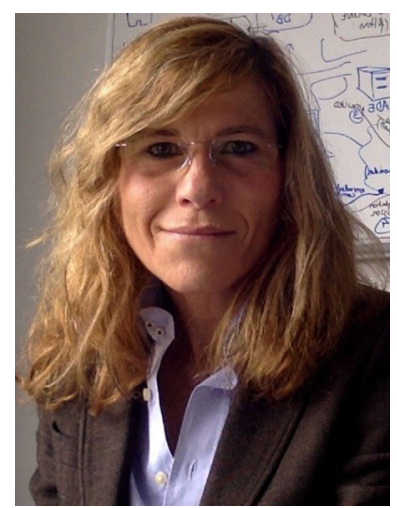

Maria Teresa Andrade holds a Ph.D. from the university of Porto in Electrotechnical and Computing Engineering. She conducts professional activities along three main axes: 1) teaching and scientific advising; 2) research; and 3) services. Teaching activities are performed within courses under the responsibility of the departments of Electrotechnical and Computing Engineering and Informatics Engineering at the University of Porto. Courses taught are normally within the field of telecommunications. Scientific advising activities are regularly performed both directly with M.Sc. and Ph.D. students as well as in co-supervision with other colleagues or with professionals in the industry. Research activities are conducted as part of her engagement at the research institute INESC Porto, where she holds the position of coordinator of the Multimedia Systems area. Research work is mostly developed within the framework of European-funded research projects, but also nationally-funded projects. The main broad area of research activity has been context-aware multimedia networking, recommendation and metadata. This activity has made possible the publication original work in international conferences and journals. Additionally, several service and research-related activities are normally carried out, notably: participation in M.Sc. and Ph.D. juries; review of scientific articles submitted to international journals and conferences; participation in technical committees of international conferences; evaluation of R\&D project proposals submitted for funding to the European Commission as well as at the national level. 